Washington L.C. dos-Santos · John David

Roberto Badaró · Luiz A.R. de-Freitas

\title{
Association between skin parasitism and a granulomatous inflammatory pattern in canine visceral leishmaniosis
}

Received: 24 August 2003/ Accepted: 10 September 2003/Published online: 11 November 2003

(C) Springer-Verlag is a part of Springer Science + Business Media 2003

\begin{abstract}
In this work we examined 76 stray dogs from an area of endemic visceral leishmaniosis, in order to determine whether the presence of skin inflammation or a specific inflammatory pattern could be taken as indicative of infection with Leishmania chagasi, and whether the parasite burden in the skin could be associated with the intensity or the nature of the inflammatory process. Inflammatory infiltrates were observed in the skin of 51 out of 55 animals with diagnosis of leishmaniosis, and in 17 out of 21 animals without signs of infection. Amastigotes were identified in the skin of 29 out of the 55 animals with diagnosis of leishmaniosis. Granuloma and a monomorphic macrophage inflammatory infiltrate, and not a mixed focal or mixed diffuse inflammation, were significantly associated with skin parasitism, both in terms of frequency $(P=0.015$ in the Chi-square test) and intensity $(P=0.005$ in the Kruskal-Wallis test). A low parasite burden was associated with a multifocal inflammatory pattern.
\end{abstract}

W. L. dos-Santos $(\square) \cdot$ L. A. de-Freitas

Centro de Pesquisas Gonçalo Moniz,

Fundação Oswaldo Cruz, Rua Valdemar Falcão 121,

Brotas, 40295-001 Salvador,

Bahia, Brazil

E-mail: wluis@cpqgm.fiocruz.br

Tel.: +55-71-3568781 ext. 210

Fax: + 55-71-3564292

W. L. dos-Santos

Escola Bahiana de Medicina e Saúde Pública,

Av. Dom João VI 274, Brotas, 40290-000 Salvador,

Bahia, Brazil

J. David

Department of Immunology and Infectious Disease,

Harvard School of Public Health,

Boston, Mass., USA

R. Badaró · L. A. de-Freitas

Faculdade de Medicina,

Universidade Federal da Bahia,

Salvador, Bahia, Brazil

\section{Introduction}

Zoonotic visceral leishmaniosis is endemic in Europe and America. The disease is caused by Leishmania chagasi and L. infantum, and is expanding into new areas (Enserink 2000), including urban centers (Silva et al. 2001). Evidence exists that $L$. infantum and $L$. chagasi are variants of the same Leishmania species (Maurício et al. 2000) and dogs have been considered their main reservoir (Dye 1996). The importance of dogs in the transmission of visceral leishmaniosis is supported by the high parasite burdens found in the skin of infected animals (Deane and Deane 1955) and the demonstration that they are readily able to infect sand flies (Travi et al. 2001).

In canine visceral leishmaniosis, the presence of amastigotes in different organs is usually observed in a background of inflammation (Martínez-Moreno et al. 1995; Tarantino et al. 2001). On the other hand, animals living in areas where the disease is endemic, particularly in tropical countries, are often subjected to arthropod bites, and to contact with other parasites that also cause inflammation in the skin (Tarantino et al. 2001). In fact, although it is recognized that dermatitis is a common finding in canine visceral leishmaniosis, the proportion of skin inflammation in dogs in areas of endemic disease that could be attributed to the presence of Leishmania is not known. Nevertheless, the characteristics of a chronic inflammation are profoundly influenced by the host's immunological response to the triggering agent (Pontesde-Carvalho et al. 2002). In human and experimental murine leishmaniosis, caused by a variety of Leishmania species, the type of the inflammatory infiltrate reflects the profile of the host immune response to the parasite (Barral-Netto et al. 1995; Lemos de Souza et al. 2000), and can be considered as morphological markers of Th1or Th2-type immune responses to Leishmania infection (Bittencourt and Barral-Netto 1995) or resistance and susceptibility to the disease (Lemos de Sousa et al. 2000). Hence, the inflammatory infiltrate in lesions of resistant 
subjects is constituted of a mixture of lymphocytes, plasm cells, and epithelioid macrophages with granuloma formation and few parasites (Lemos de Souza et al. 2000). On the other hand, in susceptible individuals, the inflammatory infiltrate is composed almost exclusively of non-activated macrophages containing large cytoplasmic vacuoles burdened with parasites, and granulomas are not formed (Bittencourt and Barral Netto 1995; Lemos de Souza et al. 2000). The type of inflammatory infiltrate in the skin of dogs with canine visceral leishmaniosis may also reflect the status of susceptibility to the disease (Ferrer et al. 1988; Fondevila et al. 1997).

In this study, the frequency and histological patterns of skin inflammation in dogs from a tropical area of endemic visceral leishmaniosis, with positive or negative laboratory tests for infection with $L$. chagasi, were investigated, aiming at identifying associations between the presence, intensity and the different patterns of inflammatory infiltrates with the presence and density of Leishmania amastigotes in the skin. Determining the inflammatory patterns associated with high and low parasite burdens in the skin may have a potential use in identifying canine immune responses associated with resistance or susceptibility to infection with $L$. chagasi/ L. infantum, as described for humans (Bittencourt and Barral-Netto 1995) and for mice (Lemos de Souza et al. 2000) with cutaneous leishmaniosis, and as suggested for dogs with visceral leishmaniosis (Fondevila et al. 1997).

\section{Material and methods}

\section{Animals}

Stray dogs (76), collected from the streets of Jacobina (an area of endemic visceral leishmaniosis, in Bahia State, Brazil), were used in this study. Diagnosis of natural infection by Leishmania was performed using the following tests: (1) culture of bone marrow, spleen or peripheral lymph node aspirate; (2) inoculation of bone marrow aspirate collected from dogs, into hamsters; (3) identification of amastigotes in skin sections (see below); (4) immunofluorescence and enzyme-linked immunosorbent assay (ELISA) for detection of specific anti-Leishmania antibodies in the serum; and (5) polymerase chain reaction (PCR) for identification of Leishmania DNA (Ashford et al. 1993, 1995). Not all the animals were subjected to all the tests. Dogs were considered infected if parasites were identified in skin sections, culture or after inoculation in hamster; or if they had both positive serological tests and positive PCR. Animals were considered negative if subjected to at least three different tests, including serology, histology and culture of bone marrow aspirate, with negative results. Using these criteria, 55 animals were considered positive and the other 21 were considered negative for Leishmania infection.

Tissue sections for histopathological analysis

Specimens of ear skin from all the animals were collected during necropsy, and fixed with formalin. Ear sections were studied because the ear is exposed to insect bites, and apparently more frequently infected in canine visceral leishmaniosis (Travi et al. 2001). Sections $(5 \mu \mathrm{m}$ thick) were stained with haematoxylin-eosin (H-E) and examined in order to define the presence or absence of inflammation, the inflammatory infiltrate pattern, and the intensity of the parasite burden. The skin was classified into the following categories: (1) normal, without inflammatory infiltrate; (2) with mixed focal inflammatory infiltrate, containing a variety of inflammatory cells including lymphocytes, macrophages and plasma cells, present as single or multiple small clusters in the skin; (3) with mixed diffuse inflammatory infiltrate, similar to the previous category, but with extensive coalescent spread of the inflammatory infiltrate through the dermis; (4) with granuloma, containing areas of epithelioid macrophages; (5) with monomorphic macrophage inflammatory infiltrate, containing clusters of non-epithelioid, vacuolated macrophages [Vichowian-like inflammatory infiltrate (see Fig. 1)]. The parasite burden was classified as follows: (1) low when rare amastigotes were seen in tissue sections, usually requiring careful search; (2) moderate when parasites were easily seen, but with restricted distribution in some macrophages in the inflammatory infiltrate; and (3) high when most of the macrophages in the inflammatory infiltrate had parasites. All the slides were examined independently by two pathologists. Results of the individual analysis were compared. The few cases in which the classifications in individual examination did not match were re-examined, in order to reach a consensus.

\section{Expression of results}

The results are expressed in tabular form as absolute numbers. The significance of the differences observed between groups was tested using the Chi-squared test or Fisher's exact probability test for cross tables using proportions. The Kruskal-Wallis test was used when differences in scores between more than two groups were compared (Glantz 1996). In all the tests the accepted level of significance corresponded to $P<0.05$.

\section{Results}

Inflammatory infiltrate in the skin of animals with and without evidence of leishmaniosis

Inflammatory infiltrates were observed in the ear of 51 out of 55 animals with diagnosis of leishmaniosis $(93 \%)$, and in the ear of 17 out of 21 animals with negative laboratory tests for the disease $(81 \%)$. This difference was not statistically significant $(P=0.206$ by Fisher's exact probability test). The distribution of the different inflammatory patterns in the skin of the animals with evidence of Leishmania infection is shown in Table 1. The difference in frequency of the different patterns of inflammatory infiltrates between infected and non-infected animals was not statistically significant $(P>0.05$ by Fisher's test).

\section{Inflammatory infiltrate and parasites in the skin}

Parasites were identified in skin sections of 29 animals: in 26 out of 53 animals with a diagnosis of leishmaniosis confirmed by other tests; in 2 for which the other performed tests (serology and PCR) were negative; and in 1 animal in which no other laboratory test was performed. The difference in the frequency of skin parasitism between animals with $(26 / 52)$ and without diagnosis of leishmaniosis, by other tests $(2 / 23)$, was statistically significant $(P<0.001$ by Fisher's test $)$. 
Fig. 1a-f Histological categories of skin in dogs from an area of endemic visceral leishmaniosis. a Normal skin. b Focal inflammatory infiltrate. c Diffuse inflammatory infiltrate. d Variety of cell types characteristic of focal and diffuse inflammatory infiltrate. e Granuloma constituted of epithelioid macrophages. f Monomorphic macrophage inflammatory infiltrate, constituted of large vacuolated macrophage containing Leishmania. (All stained with haematoxylin and eosin)
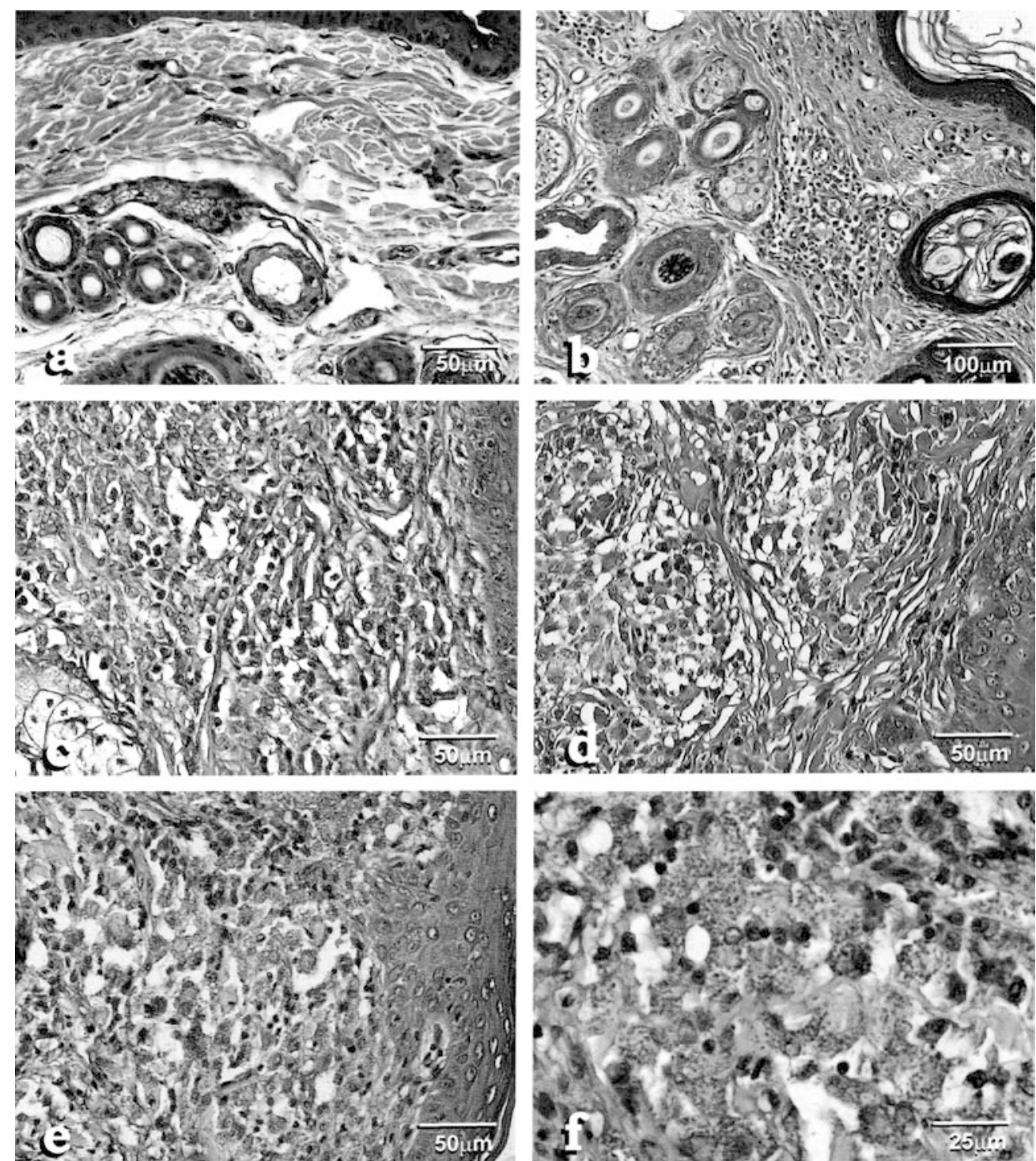

The presence of parasites in skin sections was always associated with an inflammatory infiltrate: amastigotes were visualized in 29 out of 68 animals with inflammatory infiltrate in the skin and were not observed in
8 animals without inflammatory infiltrate in the skin $(P=0.021$ by Fisher's test). The frequency of the different inflammatory patterns and their association with presence of amastigotes in the skin is shown in Table 1.

Table 1 Inflammation in ear skin of stray dogs from an area of endemic leishmaniosis with or without diagnosis of the disease

\begin{tabular}{|c|c|c|c|c|c|c|}
\hline Inflammatory pattern & \multicolumn{2}{|c|}{ Infection } & \multicolumn{4}{|c|}{ Parasite burden $^{\mathrm{a}}$} \\
\hline Focal & 6 & 24 & 22 & 6 & 2 & 0 \\
\hline Diffuse & 10 & 16 & 14 & 4 & 5 & 3 \\
\hline Granuloma & 1 & 10 & 3 & 3 & 4 & 1 \\
\hline Macrophagic $^{\mathrm{b}}$ & 0 & 1 & 0 & 0 & 0 & 1 \\
\hline
\end{tabular}

${ }^{a}$ Semi-quantitative analysis based on the following criteria: low when rare amastigotes were seen in tissue sections, usually requiring careful search; moderate when parasites were easily seen, but with restricted distribution of macrophages in the inflammatory infiltrate; and high when most of the macrophages in the inflammatory infiltrate had parasites

${ }^{\mathrm{b}}$ Monomorphic (vacuolated) macrophage inflammatory infiltrate 
Granulomatous and monomorphic macrophage inflammatory patterns were more frequently associated with the presence of Leishmania in the skin than focal or diffuse inflammatory infiltrate $(P=0.015$ by the Chisquare test). The difference in frequency of amastigotes associated either with focal or diffuse inflammatory infiltrate was not statistically significant $(P=0.167$ by Fisher's exact probability test).

Parasite burden and inflammatory pattern

Most of the animals with focal inflammatory infiltrate in the skin had no histological parasitism. Also, the parasite burden associated with focal infiltrate was of slight or moderate intensity. Diffuse and granulomatous inflammatory patterns were predominantly associated with moderate and heavy parasite burdens (Table 1). In the only animal presenting monomorphic macrophage infiltrate, the parasite burden was heavy. The difference in the parasite burdens in the skin associated with the different inflammatory patterns was statistically significant $(P=0.005$ by the Kruskal-Wallis test followed by pair wise test): parasite burdens were higher in animals with granuloma and monomorphic macrophage infiltrate than with diffuse inflammatory infiltrate $(P<0.01)$, and higher in animals with diffuse than with focal inflammatory infiltrate $(P<0.05)$.

\section{Discussion}

In this study we show that inflammatory infiltrates are a common histological finding in the skin of dogs from a tropical area of endemic leishmaniosis, irrespective of the presence of parasites or evidence of infection with Leishmania. Skin parasitism in dogs with visceral leishmaniosis was, however, always associated with inflammation. Inflammatory patterns with predominance of macrophages, such as granuloma and monomorphic macrophage infiltrates were more frequently associated with the presence of Leishmania and a higher parasite burden than pleomorphic focal or diffuse inflammatory infiltrate.

The monomorphic macrophage inflammatory infiltrate described herein is similar to the lesions of diffuse cutaneous leishmaniosis observed in human beings (Barral-Netto et al. 1995; Bittencourt and Barral-Netto 1995) and in CBA mice infected with L. amazonensis (Lemos de Souza et al. 2000), and may correspond to the nodular disease developed by dogs with ineffective cellular immune response to L. chagasi (Fondevila et al. 1997; Ferrer et al. 1988). Such an inflammatory pattern has been associated with Th2-type of immune response and susceptibility to the disease (Barral-Netto et al. 1995; Lemos de Souza et al. 2000). Presence of granuloma on the other hand, has been associated with Th1type immune response and resistance to leishmaniosis (Oliveira et al. 1993; Lemos de Souza et al. 2000; Amato et al. 2003) and to mycobacterial disease (Chensue et al.
1995). Therefore, the finding in the present work of larger numbers of amastigotes associated with granulomas than with other inflammatory patterns is apparently surprising. At least two mechanisms may explain this observation: (1) arrival of Leishmania at the skin of a immunocompetent host, either inoculated by sand flies or carried by infected macrophages (Liarte et al. 2001), initiating after its proliferation a cellular immune response with delayed-type hypersensitivity (DTH) and granuloma formation (Barral-Netto et al. 1995; Lemos de Souza et al. 2000); or (2) dissemination of large number of parasites to the skin, a possibly late event in the visceral leishmaniosis (Tafuri et al. 2001) of susceptible dogs, associated with an inflammatory granulomatous infiltrate permissive to parasite growth. In fact, evidence exists that epithelioid cells in granulomas secrete factors, such as IL-10 (Wilson et al. 1996) and a 12$\mathrm{kDa}$ protein (Mariano 1995), that inhibits macrophage and lymphocyte activation. Indeed, generation of a microenvironment with cytokines that inhibit Th1-type immune response in granulomas, favoring parasite replication, has been demonstrated in visceral murine leishmaniosis (Wilson et al. 1996). Further studies, taking into account the age of infection and the immunological status of the animals, are necessary in order to explain which of these mechanisms is responsible for the association of granuloma with the high parasite burden in the skin of dogs with visceral leishmaniosis, described in this study.

Little is known about the determinants and mechanisms involved in Leishmania location in the skin in animals with canine visceral leishmaniosis. Only a portion of dogs with visceral leishmaniosis had parasites detectable in the skin. In the present study, amastigotes were identified in the skin of $50 \%$ of the animals with serological and parasitological (including PCR) markers of natural infection with Leishmania, when using H-E stained sections. Solano-Gallego and collaborators (2001) and Travi and collaborators (2001), both using PCR for Leishmania DNA, found evidence of parasites in the skin of $70 \%$ of the animals with diagnosis of infection. Parasite species and strains (reviewed by Herwaldt 1999) and the immunological status of the host (Postigo et al. 1997) are among the factors possibly implicated in the dissemination of Leishmania to different sites, in dogs and other animals. In fact, Leishmania dissemination to the skin in dogs and human beings appears to be a late event in the course of infection (Herwaldt 1999; Travi et al. 2001). Sequential parasite dissemination has been suggested in the mouse model of visceral leishmaniosis (Wilson et al. 1996; Sulahian et al. 1997; Anstead et al. 2001). Parasites disseminate from lymph node to liver (Anstead et al. 2001) and, at a late stage only, the parasite burden increases in the spleen (Barbosa Jr et al. 1987; Wilson et al. 1996; Sulahian et al. 1997). Bertho and collaborators (1994) demonstrated that inflammation artificially induced by trauma favored the appearance of metastatic lesions of leishmaniosis in the skin of mice infected with $L$. amazonensis. In 
inflamed tissues, a range of chemokines and newly expressed adhesion molecules potentially direct the homing of infected cells (Springer 1995) and these could include Leishmania-infected phagocytes originated from internal organs. In this study, chronic inflammation was a frequent finding in the ear skin of dogs without any evidence of Leishmania infection (examined), not differing from that observed in animals with infection. Although infection cannot be completely excluded, based on the sensitivity of the tests used (Ashford et al. 1995), we may conclude that the majority of the animals in the group with negative results, and at least some of the animals in the infected group, present inflammation in the skin unrelated to Leishmania infection (Prats and Ferrer 1995). Initiation of such inflammatory processes may be attributed to a variety of conditions, for example, insect bites (frequent all year in tropical countries), trauma (Prats and Ferrer 1995) sometimes originated during fighting, and other parasitic infections (Mozos et al. 1999). The data presented herein, therefore, are consistent with the hypothesis that skin lesion precedes the arrival of L. chagasi at a site in dog's skin, and that, once there, the Leishmania induces a change in the inflammatory pattern towards the formation of granulomas (Prats and Ferrer 1995; Mozos et al. 1999) or development of a vacuolated macrophage infiltrate, that are typically found in cutaneous and visceral leishmaniosis. Alternatively, the induction of a permissive inflammatory pattern by other causes would precede the installation of Leishmania in the skin, e.g., a pre-existing inflammatory reaction with permissive granulomas would be more favorable to Leishmania growth than other inflammatory reactions (Mariano 1995; Wilson et al. 1996). In fact, those dogs with a tendency to develop this permissive inflammatory reaction would be more prone to remaining infected with Leishmania and developing high parasite burdens. Whatever the case, Leishmania-specific or non-specific immunointervention measures that would favor the healing of skin lesions or development of only focal inflammation in the skin could be useful as a means of reducing the transmission of Leishmania from infected dogs to the sand fly vector (Travi et al. 2001). Confirmation of this hypothesis requires and justifies, further studies.

Acknowledgements This work was supported by the Fundacao Oswaldo Cruz (PAPES II, grant number 250.250.320) and the Brazilian National Research Council, (CNPq, grant number 52629/96-5). We are indebted to Dr. Lain Pontes de Carvalho (Oswaldo Cruz Foundation) for his careful revision and suggestions for the text.

\section{References}

Amato VS, de Andrade HF, Duarte MI (2003) Mucosal leishmaniasis: in situ characterization of the host inflammatory response, before and after treatment. Acta Trop 85:39-49

Anstead GM, Chandrasekar B, Zhao W, Yang J, Perez LE, Melby PC (2001) Malnutrition alters the innate immune response and increases early visceralization following Leishmania donovani infection. Infect Immun 69:4709-4718
Ashford DA, Badaró R, Eulálio C, Freire M, Miranda C, Zalis MG, David JR (1993) Studies on the control of visceral leishmaniasis: validation of the Falcon assay screening test-enzymelinked immunosorbent assay (FAST-ELISA) for field diagnosis of canine visceral leishmaniasis. Am J Trop Med Hyg 48:1-8

Ashford DA, Bozza M, Freire M, Miranda JC, Sherlock I, Eulalio C, Lopes U, Fernandes O, Degrave W, Barker RH Jr, Badaró R, David JR (1995) Comparison of the polymerase chain reaction and serology for the detection of canine visceral leishmaniasis. Am J Trop Med Hyg 53:251-255

Barbosa AA Jr, Andrade ZA, Reed SG (1987) The pathology of experimental visceral leishmaniasis in resistant and susceptible lines of inbred mice. Braz J Med Biol Res 20:63-72

Barral-Netto M, Machado P, Barral A (1995) Human cutaneous leishmaniasis: recent advances in physiopathology and treatment. Eur J Dermatol 5:104-113

Bertho AL, Santiago MA, Coutinho SG (1994) An experimental model of the production of metastases in murine cutaneous leishmaniasis. J Parasitol 80:93-99

Bittencourt AL, Barral-Netto M (1995) Leishmaniasis. In: Doer W, Seifert G (eds) Tropical pathology (2nd edn). Springer, Berlin Heidelberg New York, pp 597-651

Chensue SW, Warmington KS, Ruth JH, Lincoln P, Kunkel SL (1995) Cytokine function during mycobacterial and schistosomal antigen-induced pulmonary granuloma formation. Local and regional participation of IFN-gamma, IL-10, and TNF J Immunol 154:5969-5976

Deane LM, Deane MP (1955) Leishmaniose visceral urbana (no cão e no homem) em Sobral, Ceará. Hospital 47:75-87

Dye C (1996) The logic of visceral leishmaniosis control. Am J Trop Med Hyg 55:125-130

Enserink M (2000) Infectious diseases. Has leishmaniasis become endemic in the U.S.? Science 290:1881-1883

Ferrer L, Rabanal R, Fondevila D, Ramos JA, Domingo M (1988) Skin lesions in canine leishmaniasis. J Small Anim Pract 29:381-388

Fondevila D, Vilafranca M, Ferr L (1997) Epidermal immunocompetence in canine leishmaniasis. Vet Immunol Immunopathol 56:319-327

Glantz SA (1997) Primer of biostatistics (4th edn). McGraw-Hill, New York, p 473

Herwaldt BL (1999) Lieshmaniasis. Lancet 354:1191-1199

Lemos de Souza V, Ascencao Souza J, Correia Silva TM, Sampaio Tavares Veras P, Rodrigues de-Freitas LA (2000) Different Leishmania species determine distinct profiles of immune and histopathological responses in CBA mice. Microbes Infect 2:1807-1815

Liarte DB, Mendonca IL, Luz FCO, Abreu EAS, Mello GWS, Farias TJC, Ferreira AFB, Millington MA, Costa CHN (2001) QBC for the diagnosis of human and canine American visceral leishmaniasis: preliminary data. Rev Soc Bras Med Trop 34:577-581

Mariano M (1995) The experimental granuloma. A hypothesis to explain the persistence of the lesion. Rev Inst Med Trop São Paulo 37:161-176

Martínez-Moreno A, Moreno T, Martínez-Moreno FJ, Acosta I, Hernández S (1995) Humoral and cell-mediated immunity in natural and experimental canine leishmaniasis. Vet Immunol Immunopathol 48:209-220

Maurício IL, Stothard JR, Miles MA (2000) The strange case of Leishmania chagasi. Parasitol Today 16:188-189

Mozos E, Pérez J, Day MJ, Lucena R, Ginel PJ (1999) Leishmaniosis and generalized dermodicosis in three dogs: a clinical and immunohistochemical study. J Comp Pathol 120:257-268

Oliveira GG, Santoro F, Sadigursky M (1993) The subclinical form of experimental visceral leishmaniasis in dogs. Mem Inst Oswaldo Cruz 88:243-248

Pontes-de-Carvalho L, Santana CC, Soares MB, Oliveira GG, Cunha-Neto E, Ribeiro-dos-Santos R (2002) Experimental chronic Chagas' disease myocarditis is an autoimmune disease preventable by induction of immunological tolerance to myocardial antigens. J Autoimmun 18:131-138 
Postigo C, Llamas R, Zarco C, Rubio R, Pulido F, Costa JR, Iglesias L (1997) Cutaneous lesions in patients with visceral leishmaniasis and HIV infection. J Infect 35:265-268

Prats N, Ferrer L (1995) A possible mechanism in the pathogenesis of cutaneous lesions in canine leishmaniasis. Vet Rec 134:103104

Silva ES, Gontijo CM, Pacheco RS, Fiúza VO, Brazil RP (2001) Visceral leishmaniasis in the Metropolitan Region of Belo Horizonte, State of Minas Gerais, Brazil. Mem Inst Oswaldo Cruz 96:285-291

Solano-Gallego L, Morell P, Arboix M, Alberola J, Ferrer L (2001) Prevalence of Leishmania infantum infection in dogs living in an area of canine leishmaniasis endemicity using PCR on several tissues and serology. J Clin Microbiol 39:560-563

Springer TA (1995) Traffic signals on endothelium for lymphocyte recirculation and leukocyte emigration. Annu Rev Physiol $57: 827-872$

Sulahian A, Garin YJ, Pratlong F, Dedet JP, Derouin F (1997) Experimental pathogenicity of viscerotropic and dermotropic isolates of Leishmania infantum from immunocompromised and immunocompetent patients in a murine model. FEMS Immunol Med Microbiol 17:131-138

Tafuri WL, de Oliveira MR, Melo MN, Tafuri WL (2001) Canine visceral leishmaniosis: a remarkable histopathological picture of one case reported from Brazil. Vet Parasitol 96:203-212

Tarantino C, Rossi G, Kramer LH, Perrucci S, Cringoli G, Macchioni G (2001) Leishmania infantum and Neospora caninum simultaneous skin infection in a young dog in Italy. Vet Parasitol 102:77-83

Travi BL, Tabares CJ, Cadena H, Ferro C, Osorio Y (2001) Canine visceral leishmaniasis in Colombia: relationship between clinical and parasitologic status and infectivity for sand flies. Am J Trop Med Hyg 64:119-124

Wilson ME, Sandor M, Blum AM, Young BM, Metwali A, Elliott D, Lynch RG, Weinstock JV (1996) Local suppression of IFNgamma in hepatic granulomas correlates with tissue-specific replication of Leishmania chagasi. J Immunol 156:2231-229 